\title{
Nachträge zur Bestimmungstabelle der unechten Pimeliden aus der palaearktischen Fauna.
}

\author{
Von Edm. Reitter in Paskau (Mähren). \\ Gen. Trigonoscelis Sol. \\ (Prosternalfortsatz niedergebogen.)
}

1" Die umgeschlagenen Seiten der Flügeldecken fast glatt oder einzeln punktiert, manchmal mit weitläufiger feiner Körnchenreihe; nicht gleichmäßig granuliert. Die Körnelung des Halsschildes wenig dicht und die Basis nicht ganz erreichend.

2" Scheibe des Halsschildes granuliert, Flügeldecken mit deutlichen Körner- oder Tuberkelreihen, rauh skulptiert.

3" Die Humeralreihe der Tuberkeln auf den Flügeldecken ist vorne nicht rippenartig markiert, indem die Tuberkeln daselbst nicht dichter als auf den Dorsalreihen stehen; nach hinten ist die Humeralreihe durch dichter und regelmäßiger gestellte, zugespitzte Höckerchen fast rippenartig markiert; zwischen der Humeralreihe und dem Seitenrande befindet sich nur eine einzelne Tuberkelreihe. Tarsen ohne gelbe Haarbüschel zwischen der dunklen Bewimperung.

4" Flügeldecken kurz und seitlich gerundet, ihre Oberseite mit sehr spärlichen Höckerchen besetzt, die inneren Höckerreihen schon in der Mitte erlöschend, auf den inneren zwei primären, meist wenig ordentlichen Reihen nur etwa fünf Tuberkeln vorhanden. Die umgeschlagenen Seiten der Flügeldecken kahl.

5" Die Tuberkeln auf den Flügeldecken wenig groß, auf den Reihen auch vorne schwach abgeflacht, die inneren Reihen zur Naht schwächer werdend, die erste primäre Reihe neben der Naht nur spitz körnchenförmig ausgeprägt. - Transcaspien: Krasnowodsk; Tekke. — T. grandis Gebl. Kr. Fst., non Falderm. Reitt. T. 25, $34=$ Schrenlii var.? corallifera Reitt.

5’ Die Tuberkeln auf den Flügeldecken sind sehr groß, meist unregelmäßig gestellt, die vorderen der Scheibe stark abgeflacht und glänzend, die inneren Reihen sind nicht schwächer ausgeprägt und die erste primäre an der Naht so wie die anderen, meist nur aus wenigen Pusteln bestehend. Große Art. Long. 27-31 mm. - Buchara, Kaschgar; Kirghisia nach Gebler. - Tr. pustulifera Reitt. i. lit.

Schrenki Gebler.

Wiener Entomologische Zeitung, XXVI. Jahrg., Heft III (31. März 1907). 
4' Flügeldecken ein wenig länger und mehr parallel, ihre Oberseite mit ziemlich dicht gestellten, mehr regelmäßigen, bis zum Absturz reichenden Tuberkelreihen, auf den inneren zwei primären Reihen etwa 8--10 oder noch mehr Höckerchen vorhanden,

6" Die umgeschlagenen Seiten der Flügeldecken auf ihrer vorderen Hälfte äußerst fein, wenig dicht, fast staubartig behaart. Long. 22-25 mm. -- Transcaspien.

nodosa Fisch.

6' Die umgeschlagenen Seiten der Flügeldecken, außer den einzelnen feinen, aus den Körnchen entspringenden Härchen, kahl. Long. 28-32 mm. - Transcaspien. v. gigas Reitt.

$3^{\prime}$ Die Humeralreihe der Tuberkeln ist bis zur Schulterecke dichter gereiht und auch vorne deshalb etwas rippenartig markiert. Körper etwas gestreckter, mit weniger großen, oben nicht so deutlich abgeflachten Tuberkelreihen.

7" Der umgeschlagene Teil der Flügeldecken ist ziemlich dicht und sehr fein, fast reifartig gelblich behaart; Scheibe ziemlich dicht und kräftig gehöckert. Tarsen ohne goldgelbe Haarbüschel.

8" Spitze der Flügeldecken dicht und fein behaart. -- Long. 22-26 mm. - Südrußland, bei Astrachan. - Icon. pg. 48, I. C., Fig. 14.

muricata Pallas.

8' Spitze der Flügeldecken, außer den Härchen in den Punkten und Härchen, fast kahl. Vielleicht nur eine Form der vorigen. Sie ist gewöhnlich etwas größer. - Long. 24-28 mm. Transcaspien. - Ins. Lehmann 1847, 5.

gemmulata Mén.

Eine kleinere Form mit viel spärlicheren Höckerreihen, wovon die inneren gleichzeitig schwächer sind und bei welcher auch die Humeralreihe weiter vorne wenig dicht ist, kommt bei Kurutsch vor. - Reitt. Tab. 25. 233. v. sparsa Reitt.

7' Der umgeschlagene Teil der Flügeldecken ist fast kahl, weitläufig fein punktiert und mit einzelnen sehr feinen Härchen. Wenigstens das zweite, dritte und vierte Glied der Mittel-, oft auch der Hintertarsen auf der Spitze ihrer Unterseite mit einem kleinen goldgelben Haarpinsel.

10" Scheibe des Halsschildes in der Mitte im weiten Umfange flach gekörnt, die Körnchen verwischt, flach, matt, undeutlich. Flügel- 
decken kurz, mit kleinen Höckerchen, diese nur mit einem sehr kurzen, nach hinten gerichteten Haare. Long. 26 -29 mm. - Taschkend, Turkmenien, Buchara. T. 25,236 .

sublaevicollis Reitt.

10' Körnchen des Halsschildes meistens deutlich erhaben, normal. Körnchen der Flügeldecken länger behaart.

11" Die Mitteltarsen mit kleinem, die Hintertarsen oft mit undeutlichem oder fehlendem gelben Haarpinsel auf ihrer Unterseite.

12" Hinterwinkel des Halsschildes, von oben gesehen, wegen der von oben nicht sichtbaren Ausschweifung nur rechteckig oder etwas stumpfwinkelig erscheinend. Hinterschienen innen mit Borstenzähnchen besetzt.

13" Oberseite glänzend, die Körnchen auf den Flügeldecken rundlich, tuberkelartig, ziemlich kräftig, vorne sind dieselben auf der inneren Scheibe abgestumpft, glänzend, an ihrem Apicalrande nur mit mäßig langen Haaren besetzt.

a"' Spitze der Flügeldecken nur mit ganz kleinen Körnchen, gleichwie sie auch auf der Scheibe zwischen den großen Reihenhöckern stehen. Die Scheibe zwischen der Naht und Humeralrippe mit fünf regelmäßigen Tuberkelreihen, dazwischen nur mit sehr feinen Körnchen am Grunde. Vorderschienen zur Spitze verbreitert, der Apicalzahn auf der Außenseite normal, etwas nach einwärts gedrückt, nicht deutlich nach außen gerichtet. Long. 21-31 mm. -- Transcaspien, Turkestan (Margelan, Taschkent, Buchara). - Reitt. Tab. 25, 234 und 237.

Zoufali Reitt.

a" Spitze der Flügeldecken mit viel größeren und dichter gestellten Körnern als sie zwischen den regelmäßigen fünf Tuberkelreihen auf der Scheibe stehen. Vorderschienen mit einem nach außen gerichteten Endzahn. Long. 21-30 mm. - Transcaspien: Tschingan.

v. apicalis nov.

a' Spitze der gedrungenen Flügeldecken mit ganz kleinen Körnchen; die Tuberkeln der primären Reihen kräftig, oben glänzend, die sekundären ebenso groß wie die primären, weshalb die Reihen zahlreicher, aber besonders hinten konfus erscheinen. Vorderschienen normal wie bei der Stammform. Beine sehr kräftig. Long. 27-30 mm. -- Transcaspien. - Rttr. T. 25, 235, 
13’ Etwas glänzend, Flügeldecken matt, glanzlos, die kleineren Körner auf den länglichen Flügeldecken in regelmäßigen Reihen gestellt, alle scharf konisch zugespitzt, die sekundären Reihen von der Mitte zur Spitze vorhanden, alle regelmäßig gestellt, aus gleich großen Körnern bestehend; die Spitze der Flügeldecken dicht und kräftig gekörnt. Die Haare am Hinterrande der Körner der Decken lang, schwarz.

b" Die Körner der Reihen auf den gestreckten Flügeldecken sind auf der Scheibe wenig dicht gestellt, recht fein und scharfeckig. Endzahn der Vorderschienen normal, leicht zurückgestellt. Long. $26-31 \mathrm{~mm}$. - Samarkand.

v. seriatulus nov.

b' Die Körner der Reihen auf den gedrungenen Flügeldecken sind sehr dicht gestellt, fein und scharfeckig, etwas kräftiger als bei der vorigen Form. Endzahn der Vorderschienen etwas nach außen gestellt. Long. 27 mm. - Turkestan: Sefid-kuh.

\section{v. aequalis nov.}

12' Halsschild von oben gesehen mit scharf rechteckigen oder fast spitzigen Hinterwinkeln, diese wegen der vor ihnen auch von oben übersehbaren Ausschweifung etwas nach außen gerichtet. Die länglichen, vorn parallelen Flügeldecken nur mit feinen Körnchenreihen, die Körnchen hinten mit sehr langem Haare. Hinterschienen kurz beborstet, innen ohne Borstenzähnchen. Der umgeschlagene Rand der Flügeldecken deutlich punktiert. Long. 25-30 mm. - Margelan, Namangan.-D. 1882, 95.

submuricata $\mathrm{Kr}$.

11' Die Mittel- und Hintertarsen mit auffälligem, großem goldgelben Haarbüschel an den Spitzenrändern ihrer Unterseite. Oberseite mit kleinen, am Halsschilde abgeflachten, auf den länglichen Flügeldecken in fünf weitläufig gekörnten Reihen gestellten Körnerreihen; Humeralrippe dicht und fein gekörnt, die Körner hinten mit sehr langen, leicht deflorierbaren schwarzen Haaren besetzt. Körper groß und gestreckt; der Apikalzahn der Vorderschienen leicht nach außen vortretend. - Long. 24-30 mm. - Turkestan: Margelan, Buchara, Alai. Reitt. Tab. 25, 236.

fasciculitarsis Reitt.

2' Die Scheibe der ganzen Oberseite des Körpers bei oberflächlicher Besichtigung glatt erscheinend, nur die Spitze der Flügeldecken ist deutlicher granuliert. Vorderschienen mäßig verbreitert, mit nach außen vortretendem Endzahne. 
15" Schenkel wie gewöhnlich gekörnt. Flügeldecken eiförmig, mit feiner, aber deutlicher, dicht gekörnelter Humeralrippe, die Scheibe sehr fein spärlich gekörnelt, hinten mit zwei bis drei deutlicheren Körnchenreihen. Long. 23-26 mm. -- Chinesisch Turkestan, Andischan, centrale Mongolei. - Horae 1887, 529 .

sublaevigata Reitt.

15’ Schenkel dicht und stark punktiert. Flügeldecken länglich eiförmig, mit sehr vereinzelten, reihig gestellten, wenig auffälligen Raspelkörnchen, ohne Humeralrippe, diese durch eine Reihe von weitläufigen gereihten Körnern substituiert. Long, 21-25 mm. - Chinesisch-Turkestan: Aga-Buluk, Taksun, Chami. - Trigonocnemis Holdereri Reitt. W. 1900, 161.

Holdereri Reitt.

1' Die umgeschlagenen Seiten der Flügeldecken ziemlich dicht und kräftig granuliert. Kleine Arten.

16" Halsschild wenig dicht, flach granuliert, die Körnchen erreichen nicht die Basis. Mittelbrust gewölbt, vorn senkrecht abfallend. Scheibe der kurz eiförmigen Flügeldecken ziemlich dicht und in wenig ordentlichen Reihen granuliert, die Körnchen hinten wie gewöhnlich zugespitzt und mit einem schwarzen Haar versehen. Long. 18-21 mm. - Transcaspien. -- Tr. callosa Motsch., seriata Fist., simuatocollis Desbr. echinata Fisch. Alld.

16' Halsschild gedrängt und bis zum äußersten Vorder- und Hinterrande granuliert.

17" Basalrand des Halsschildes äußerst schmal, gleichmäßig, Hinterwinkel rechteckig. Mittelbrust horizontal, vorne plötzlich senkrecht abfallend. Flügeldecken zwischen der Naht und der Humeralreihe mit zwei mehr prononcierten Tuberkelreihen, dazwischen mit größeren und kleinen Körnern besetzt. Nitteltarsen mit drei, Hintertarsen mit zwei kleinen gelben Haarpinseln versehen. Long. $14-20 \mathrm{~mm}$. - Transcaspien, Turkestan: Margelan, Alai, Namangan. - D. 1882, 88.

planiuscula $\mathrm{Kr}$.

17' Basalrand des Halsschildes schmal, glatt, an den Seiten verbreitert, Hinterwinkel stumpf. Die Mittelbrust schräg im sanften Bogen nach vorne abfallend. Flügeldecken in dichten, gleichmäßigen Reihen granuliert, dazwischen feine Körnchen am Grunde. Tarsen ohne deutliche gelbe Haarpinsel. Long. $20-30 \mathrm{~mm}$. Russisch-Armenien: Ordubat.

armeniaca Fald. 


\section{Gen. Sternoplax Friv.}

(Prosternalfortsatz hinter den Vorderhüften verlängert.)

1" Prosternalspitze kurz, am abschüssigen Teile vorgebogen, die Spitze ringsum dicht gelb tomentartig behaart.

2" Der umgeschlagene Rand der Flügeldecken ist punktiert und nur mit wenigen feinen Körnchen besetzt. Halsschild mit spärlichen flachen Körnchen, die Hinterwinkel stumpf begrenzt. Flügeldecken punktiert und die Scheibe mit sehr feinen raspelartigen, weitläufigen Körnchen reihig besetzt. Bauch mit gedrängten groben und feinen Punkten runzelig zerstochen, nur mit spärlichen dunklen Härchen besetzt. - Long. 18-20 mm. Astrachan, Ural, Kirghisia, Transcaspien, Aulie-Ata. - Tr. Perevostchiliowi Zubk. - B. M. 1832, V, 130.

\section{deplanata Kryn.}

2' Der umgeschlagene Rand der Flügeldecken mäßig dicht granuliert. Halsschild ziemlich dicht mit flachen Körnern besetzt. Halsschild mit rechteckig begrenzten Hinterwinkeln.

3" Der umgeschlagene Rand der Flügeldecken mit runden einfachen Körnchen wenig dicht besetzt, Scheibe der Flügeldecken zwischen der Naht und der gekörnelten Schulterrippe auf glattem Grunde wenig dicht gekörnt, die Körner vorn wenig spitzig, mehr weniger gereiht, die Körner zur Naht und Spitze feiner werdend. Long. 17 -20 mm. - Buchara, Sefid-kuh, Transcaspien: Penschdeh. - Ins. Lehmann II, 6, Taf. 3, Fig. 6.

seriata Mén.

3' Der umgeschlagene Rand der Flügeldecken mit kräftigen raspelartig zugespitzten Körnern besetzt, Scheibe der Flügeldecken zwischen der Naht und der dicht gezähnelten Humeralrippe mit dichten, reibeisenartigen ungleichen Höckerchen und Körnern zusammengedrängt, dazwischen auch mit Punkten und kleinen Körnchen durchsetzt, so daß der Grund damit fast ganz ausgefüllt erscheint, die Körner nicht deutlich gereiht, nur hinten vor dem Absturze zwei kurze Längsreihen schlecht angedeutet. Long. 18-20 mm. - Kirghisen-Steppe, Transcaspien, Turkestan, Ost-Persien, selten. -- B. M. 1833, 227.

affinis Zubk.

1' Die Prosternalspitze ohne hellen, tomentartigen, strahlenförmigen Haarsaum. 
4" Flügeldecken oval, samt den Seiten mehr weniger gewölbt, die Humeralrippe nicht prononciert, den Hinterleib nicht von der Unterseite kantig abschließend, die Seiten der Flügeldecken von der feinen Humeralrippe nicht senkrecht nach unten abfallend. Körper Ocnera-artig.

5" Oberseite außer den schwarzen Höckerhaaren kahl, Unterseite fein, meist dunkel behaart oder fast kahl, nicht tomentiert, Halsschild und Flügeldecken ohne große, glatte Pusteln.

6" Schenkel stark und dicht punktiert; Bauch mit großen, dichten, ganz flach abgeschliffenen Körnern besetzt, glänzend. Ziemlich groß, schwarz, glänzend, Halsschild nur an den Seiten mit verwischten, undeutlichen Tuberkeln, auf der Scheibe glatt, Flügeldecken mit fein gezähnter Humeralreihe, zwischen dieser und der Naht mit fünf Reihen weitläufig gestellter, kleiner Raspelkörnchen, dazwischen noch mit einzelnen kleineren besetzt. Oberseite oberflächlich fast glatt und kahl aussehend. Long. 19-27 mm. - Namangan, Alai, Margelan. - D. 1882, 87.

laeviuscula $\mathrm{Kr}^{1}{ }^{1}$ )

6' Schenkel granuliert. Bauch dicht gekörnt, matt.

7" Flügeldecken länglich oval, mit gereihten Körnern dicht besetzt, ohne glatte Dorsalrippen, zwischen der Naht und der dicht gekörnten Humeralreihe mit zwei etwas stärker vortretenden dorsalen Körnerreihen, dazwischen meist noch eine sekundäre, wenig auffällige, in der Mitte und zwischen diesen noch mit einer gleichartigen tertiären, die Körnchen gegen die Naht zu feiner, alle mit ziemlich kurzem schwarzen Haare; die Seiten der Flügeldecken zwischen der Humeralrippe ebenfalls dicht gekörnt, der umgeschlagene Seitenrand sehr fein und weitläufig gekörnelt. Halsschild ziemlich dicht mit rundlichen Körnern besetzt, vor der fast geraden Basis mit schmaler, glatter Fläche; Hinterwinkel fast rechteckig oder etwas stumpf. Prosternalspitze nur schwach gerundet vorgezogen. Prosternum gekörnt, die mittleren Tarsen wie gewöhnlich mit vier, die hinteren mit drei gelben Haarpinseln auf der Unterseite. Vom Aussehen einer schlanken, kieineren Ocnera. Long. $19 \mathrm{~mm}$. - MittelPersien; von Herrn A. Matthiessen gesammelt.

\section{Matthiesseni n. sp.}

1) Dieser Art ähnlich ist die mir unbekannte Tr. Reitteri Csiki (Zichy, Reise 1901, 111) aus der Mongolei. Die Tarsen haben gelbe Haarpinsel. 
Der vorigen Art sehr ähnlich, aber der Thorax weniger dicht gekörnt, die Basis in der Mitte deutlich ausgebuchtet, Flügeldecken kürzer oval, etwas gewölbt, die Körnchen weniger dicht gestellt und kleiner, die Körnchen der drei Dorsalreihen deutlicher erhaben, stärker prononziert, die tertiären kaum als Reihen erkennbar, die Borstenhaare der Tuberkeln sind ganz kurz, Behaarung der Beine wie dort rostrot. Long. 18-22 mm. - Semiretschié: Djarkent, Flußgebiet des Ili, im April 1906 von Herrn G. Souvorow gesammelt. Souvorowiana n. sp.

7' Flügeldecken auf mattem, fein gekörneltem Grunde mit zwei glatten, glänzenden Dorsalrippen, diese hie und da mehr weniger kerbartig unterbrochen. Auch die Naht ist teilweise erhöht. Humeralkörnchenreihe deutlich, auch die Seiten von der Humeralreihe zum Seitenrande granuliert; alle Zwischenräume zwischen den Rippen nach hinten mit einer verkürzten deutlicheren Körnchenreihe. Halsschild mit gedrängten, groben, flachen, auf der Scheibe ganz abgeschliffenen Körnern besetzt, diese den Vorder- und Hinterrand erreichend. Mittelbrust etwas nach vorne geneigt, dann plötzlich senkrecht abfallend und buckelig; Prosternumspitze mäßig gerundet vorgezogen. Long. 14-19 mm. - Mongolei. - Termesz. 1889, 207.

Szechenyi Friv.

5' Oberseite mit feinem dichten, weißen oder gelben Toment fleckig besetzt, die Unterseite samit den Fühlern und Beinen in gleicher Weise hell tomentiert, die Schenkel nur spärlich gekörnelt. Halsschild auf mattem Grunde mit sehr großen, dicht stehenden, abgefiachten, glänzenden Tuberkeln besetzt; die Flügeldecken ebenfalls mit großen, glänzenden, abgeschliffenen Höckern auf mattem, sehr fein und spärlich gekörneltem Grunde. Das helle Toment verteilt sich auf der Oberseite; zwei kleine Schrägflecken vorne am Halsschilde, zwei größere Schrägmakeln vor der Basis; Flügeldecken an der Basis nach außen breiter tomentiert, an der Spitze stehen mehrere Längs- und Schrägflecken. Beine zart. Long. 16-19 mm. - Ost-Turkestan, Kaschgar, Thibet. - Trig. pustulosa Reitt. T. 25, 243. - Cist. Ent. II, 1879, 475; Rev. Russ. 1903, 100 . lacerta F. Bates.

4' Flügeldecken kurz gebaut, oben mehr weniger stark abgeflacht, mit prononzierter Humeralrippe, diese begrenzt die abgeflachte Scheibe nach innen und die steil abfallenden Seiten nach außen; Schulterwinkel stark nach vorne gezogen. Körper Diesia-artig. 
S" Schwarz, glänzend, Flügeldecken auf mattem, spärlich und sehr fein gekörneltem Grunde mit glatten, glänzenden, hoch erhabenen, nur hinten gekerbten Rippen, im ganzen fünf, je zwei dorsale und eine gemeinschaftliche suturale. Halsschild fast glatt, nur an dèn Seiten gekörnt, vor der Basis mit tiefem Quereindrucke. Prosternumspitze kurz gerundet vorgezogen. Mittelbrust buckelig gewölbt. Long. $18-20 \mathrm{~mm}$. - Thibet: Kuku-noor. D. $1899,204$.

costatissima Reitt.

8' Flügeldecken ohne glatte, glänzende Rippen, höchstens mit feinen Körnchenreihen.

$9^{\prime \prime}$ Die schmalen Vorderschienen auf ihrer Außenseite mit einigen ungleichen, sehr langen, (6--8) nagelartigen Zähnchen besetzt, Prosternum hinter den Hüften eine Strecke herabgebogen und in der Vitte des Abfalles als spitziges Höckerchen vortretend. Halsschild mit normaler Granulierung, Flügeldecken länglich oval, oben flach, zwischen der Naht und der dicht gezähnelten Humeralrippe mit zwei primären dorsalen Körnerreihen, diese hinter der Mitte etwas rippenförmig erhöht, hinten abgekürzt, die inneren schwächer ausgeprägt, die Scheibe dazwischen etwas konkav; sekundäre gereihte Körner sind vorhanden, dazwischen am Grunde sehr fein gekörnt und einzeln punktiert. Bauch dicht granuliert. Körper schwarz, glänzend. Long. 15-17 mm. - Aulie-Ata. - D. 1901, 78.

auliensis Reitt.

9' Die Vorderschienen zur Spitze breiter, am Außenrande gekerbt oder dicht und fein gezähnelt.

10" Mittelbrust mit horizontaler Beule oder gehöckert, von da nach vorne senkrecht abfallend.

11" Prosternalfortsatz kurz, am Ende abgerundet oder abgestumpft, hinter den Hüften leicht niedergebogen und dann kurz horizontal verflacht.

12" Bauch fein behart, auf punktiertem und hautartig oder lederartig skulptiertem Grunde mit zahlreichen kleinen Körnchen besetzt. Endsporne der Vorderschienen fast von gleicher Länge. Halsschild granuliert, mit querem Basal- und flachem Discoidaleindruck, Flügeldecken flach, dicht und fein gekörnt, die Körnchen zur Naht feiner werdend, nur zwei angedeutete Discoidalreihen zwischen den Körnchen erkennbar. Long. 15-18 mm. Turkestan (Samarkand), A lai, Nördliche M on gol ei: KurutschDagh, Kaschgar. - Reitt. Tab. 25, $241 . \quad$ juvencus Reitt. 
12' Bauch fein behaart, von kleinen, mittleren und groben Punkten sehr dicht zerstochen, ohne erhabene Körnchen dazwischen. Enddorne der Vorderschienen sehr ungleich, der längere stärker gebogen.

13" Prosternalende stumpf zugespitzt. Beine und Tarsen dunkelbraun behaart. Oberseite matt. Die Körnchen der Deckenscheibe mit geneigtem, schwarzem, wenig langem Haar, dazwischen noch mit längeren Haaren dünn besetzt. Sonst der nächsten Art ähnlich, aber größer. Long. 16-21 mm. -- Süd-Turkestan, Mongolei. - Platyope grandis Fald. Mém. Ac. Petrsbrg. II, 387 (1835). - - ? Tr. setosa Bates, Cist. 1879, 475. grandis Fald.

13' Prosternalende elliptisch abgerundet. Beine und Tarsen braunrot behaart. Oberseite glänzend. Halsschild deutlich, in der Mitte feiner granuliert, Basis fein gerandet. Flügeldecken abgeflacht, oben wenig dicht spitzig, zur Naht feiner gekörnt, davon zwischen der Naht und der dicht gekerbten Humeralkante mit zwei angedeuteten Körnchenreihen, die Seiten zwischen der Humeralrippe und dem Seitenrande vorne mit kurzer Körnerreihe. Die Körnchen der Scheibe nur mit sehr kurzem, schwarzem, anliegendem Haare. Long. 16-18 mm. - Kaschgar. kashgarensis n. $\mathrm{sp}$.

11' Prosternalfortsatz länger, von den Mittelhüften gerade, horizontal nach hinten verlängert, hinter den Hüften nicht niedergebogen, am Ende mehr weniger abgerundet. Bauch gedrängt, ungleich punktiert, nicht granuliert. Halsschild mit schwacher Querfurche vor der Basis und einer flachen Discoidaldepression. Körper und Skulptur wie bei den vorigen. Flügeldecken mit schwarzen, kurzen, stark geneigten und längeren aufgerichteten Haaren auf der Scheibe.

14" Oberseite matt, Flügeldecken und ihr umgeschlagener Rand am Grunde glatt, nicht chagriniert, Halsschild außer den schwarzen Härchen an der Spitze der Tuberkeln ohne helle Zwischenbehaarung. Long. 14-22 mm. -- Samarkand, Namangan. - Reitt. Tab. 25, 242. impressicollis Reitt.

14' Oberseite glänzend, Flügeldecken (besonders hinten) und ihr umgeschlagener Rand hautartig chagriniert, Halsschild außer den dunklen Körnerbörstchen mit feiner, wenig dichter, greiser Zwischenbehaarung. Long. $20 \mathrm{~mm}$. -- Mongolei: Oase Nia. - Hor. 1887, 377.

niana Reitt.

10' Mittelbrust nach vorne allmählig im Bogen sanft abfallend, ohne vorstehende Beule. 
15" Prosternum hinter den Hüften leicht niedergebogen, sehr kurz, stumpf zugespitzt. Die Seiten der Flügeldecken zwischen der Humeralrippe und dem Seitenrande auf der vorderen Hälfte stark granuliert. Flügeldecken scharf, zur Naht schwächer, an der Spitze stärker granuliert, zwischen der Naht und der dicht gehöckerten Humeralrippe mit zwei deutlicheren Körnerreihen. Unterseite samt den Fühlern und Beinen dicht und fein gelblich tomentiert, auch der Kopf fein behaart.

16" Oberseite etwas glänzend. Bauch dicht gelblich tomentiert, mit Kahlpunkten, am Grunde dicht und sehr fein punktuliert, mit einzelnen deutlicheren Punkten dazwischen, Mittelbrust stark, Prosternum und Hinterbrust fein granuliert. Halsschild mit ziemlich großen und dicht gestellten, nur längs der Mitte feineren Körnern besetzt. Long. 17-20 mm. - Mongolei: Kan-ssu. - Term. 1889, 206.

Kraatzi Friv. ${ }^{1}$

16' Ober- und Unterseite ganz matt. Bauch dicht gelblich tomentiert, ohne deutliche Kahlpunkte, am Grunde glatt, matt, nur mit schwer erkennbaren zerstreuten Pünktchen besetzt; Mittelbrust und Prosternum kaum, Hinterbrust fein granuliert. Halsschild an den Seiten und in der Mitte fein, dazwischen etwas stärker granuliert. Flügeldecken etwas breiter und stärker abgeflacht. Long. 18 mm. Wüste Gobi, 28. Mai 1898 von Dr. Holderer mitgebracht und vorher als Kraat: $i$ angesprochen. opaca n. sp.

15' Prosternum von den Mittelhüften gerade horizontal nach rückwärts verlängert, wenig lang, am Ende elliptisch abgerundet.

17" Nur die Unterseite dicht gelblich tomentiert, Vorder-, Mittelund Hinterbrust granuliert, Bauch ohne Körnchen, Oberseite fast kahl, schwarz, etwas glänzend, Halsschild ziemlich dicht, in der Mitte feiner und spärlicher granuliert, Flügeldecken flach gewölbt, die Humeralecke stumpf und wenig prononziert, Scheibe sehr fein und spärlich, nach innen feiner granuliert, oberflächlich fast glatt aussehend, am Grunde lederartig gerunzelt, Humeralrippe dicht granuliert, Spitze mit stärkeren Körnern versehen, der Raum zwischen Humeralrippe und Seitenrand dicht und grob granuliert. Der umgeschlagene Seitenrand dichter und stärker als bei den verwandten Arten gekörnt.

1) Mit dieser Art und Seidlits $i$ vergleicht Csiki in Zichys Reise 1901, 110, seine Tr. Zichyi Csiki aus der Mongolei, die nach der unzulänglichen Beschreibung hier nicht eingereiht werden kann.

Wiener Entomologische Zeitung, XXVI. Jahrg., Heft III (31. März 1907). 
Mitteltarsen mit kleinen gelben Haarpinseln. Long. $18-19 \mathrm{~mm}$. - Iongolei: Kan-ssu. - Horae 1889, 6 d5.

mongolica Reitt. $\left.{ }^{1}\right)$

17' Unterseite dicht und fein, Oberseite weniger dicht greis tomentiert, Vorder- und Mittelbrust fein, die Hinterbrust kaum, der Bauch nicht gekörnt. Schwarz, fast matt, Fühler und Beine dünn und lang, Glied 4-8 sehr gestreckt, Halsschild schwach quer, ziemlich fein granuliert, Flügeldecken ganz wie bei Diesia, oben abgeflacht, neben der fein gezähnelten Schulterrippe etwas konkav, sehr spärlich, zur Naht fast erloschen gekörnt, zwischen Naht und Schulterrippe zwei Reihen dichterer Körnchen, Seiten der Flügeldecken nur am oberen Rande spärlich granuliert, umgeschlagener Rand spärlich und sehr fein gekörnelt, tomentiert. Vorderschienen mit zirka acht längeren Dornzähnchen, die hinteren vier Tarsen beiderseits lang fuchsrot behart, ohne deutliche gelbe Harpinsel in der Mitte ihrer Unterseite: Vordertarsen unten lang behaart. Long. 20-22 mm. - Kuldscha. D. 1901,179 .

Iduna Reitt.

1) Siehe auch die mir jetat nicht vorliegende Tr. Seidlitri Reitt. T. 25, 243, aus der Mongolei.

\section{Notiz iiber Liodes nitidula Er.}

Von Liodes nitidula enthielt ich ein Exemplar vom Ivan (BosnaHerzegovina), bei welchem die Glieder 7,8 und 9 der Fühlerkeule fast quadratisch sind, im Gegensatz zu Individuen aus Croatien, bei denen dieselben Glieder stark quer sind. Da L. Discontignyi Bris. aus den Pyrenäen sich hauptsächlich durch dieses Merkmal von nitidula unterscheidet, wären mir Exemplare von nitidula und ähnlichen Arten, namentlich aus den Pyrenäen behufs Vornahme eines genauen Vergleiches sehr erwünscht!!

Dr. A. Fleischer.

\section{Dipterologische Notiz.}

Herr Prof. Strobl hatte die Güte jene Dipteren zu determinieren, die sich bei meiner Zucht der Liodes cimammomea aus Tuber brumale (Herbsttrüffel) mitentwickelten. Es sind dies Tephrochlamys flavipes Zett. und Sciara macilenta Wintz., beide sind häufige Arten. Dr. A. Fleischer. 


\section{$2 \mathrm{BHL}$ Biodiversity Heritage Library}

Reitter, Edmund. 1907. "Nachträge zur Bestimmungstabelle der unechten Pimeliden aus der palaearktischen Fauna." Wiener entomologische Zeitung 26, 81-92. https://doi.org/10.5962/bhl.part.5118.

View This Item Online: https://www.biodiversitylibrary.org/item/44122

DOI: https://doi.org/10.5962/bhl.part.5118

Permalink: https://www.biodiversitylibrary.org/partpdf/5118

\section{Holding Institution}

Smithsonian Libraries

\section{Sponsored by}

Smithsonian

\section{Copyright \& Reuse}

Copyright Status: NOT_IN_COPYRIGHT

This document was created from content at the Biodiversity Heritage Library, the world's largest open access digital library for biodiversity literature and archives. Visit BHL at https://www.biodiversitylibrary.org. 